\title{
BMJ Open Association between sepsis and retinopathy of prematurity: a systematic review and meta-analysis
}

\author{
Xiaofen Wang, ${ }^{1}$ Kun Tang, ${ }^{1,2}$ Ling Chen, ${ }^{1}$ Sixiang Cheng, ${ }^{1}$ Huilan $\mathrm{Xu}^{1}$
}

To cite: Wang $X$, Tang $\mathrm{K}$, Chen $\mathrm{L}$, et al. Association between sepsis and retinopathy of prematurity: a systematic review and meta-analysis. BMJ Open 2019;9:e025440. doi:10.1136/ bmjopen-2018-025440

- Prepublication history and additional material for this paper are available online. To view please visit the journal (http:// dx.doi.org/10.1136/bmjopen2018-025440).

Received 19 July 2018 Revised 19 February 2019 Accepted 29 April 2019

Check for updates

(c) Author(s) (or their employer(s)) 2019. Re-use permitted under CC BY-NC. No commercial re-use. See rights and permissions. Published by BMJ.

${ }^{1}$ Department of Social Medicine and Health Management, Xiangya School of Public Health, Central South University, Changsha, Hunan, China ${ }^{2}$ Department of Research, Xiangya Hospital, Central South University, Changsha, Hunan, China

Correspondence to Professor Huilan Xu; xhl1120131@163.com

\section{ABSTRACT}

Objective To explore the association between sepsis and retinopathy of prematurity (ROP) in premature infants.

Design A systematic review and meta-analysis.

Data sources We performed a systematic search of PubMed, the Cochrane Library and Embase from 1 January, 2000, to 1 January, 2018, with no language restrictions and reported the relationship between sepsis and ROP.

Eligibility criteria Original observational studies, including cohort studies and case-control studies.

Data extraction and synthesis Two reviewers independently completed the study selection and data extraction. The OR and corresponding 95\% $\mathrm{Cl}$ were used to measure the risk of sepsis in patients with ROP. The heterogeneity between studies was evaluated using Cochran's $Q$ test and the $\mathrm{I}^{2}$ statistic. The Newcastle-Ottawa Scale was adopted to evaluate the quality of each of the included studies, and the Grading of Recommendations Assessment, Development and Evaluation approach was used to assess the quality of the evidence.

Results Sixteen studies with a total sample size of 12466 premature infants and 2494 cases of ROP were included in this meta-analysis. Adjusted analysis showed that sepsis was closely related to any stage of $\mathrm{ROP}(\mathrm{OR}=1.57$, $95 \% \mathrm{Cl} 1.31$ to 1.89$)$ and severe stage of ROP $(\mathrm{OR}=2.33$, $95 \% \mathrm{Cl} 1.21$ to 4.51 ) in premature infants, with $56.3 \%$ and $81.8 \%$ heterogeneity, respectively. Subgroup analyses showed that heterogeneity was obvious in prospective cohort studies $\left(l^{2}=62.1 \%, p<0.001\right)$. In a sensitivity analysis, we found that removing any single study did not significantly change the overall effect value. The quality of the evidence was rated as low for both any stage of ROP and severe stage of ROP.

Conclusions Sepsis increases the risk of ROP in preterm infants. However, considering that all included studies are observational and causality can rarely be established, additional evidence is needed to substantiate this finding and provide advice for practice.

\section{INTRODUCTION}

Retinopathy of prematurity (ROP) is a vascular proliferation eye disease that affects premature infants and always leads to visual impairment, strabismus and blindness. ${ }^{1}$ ROP has emerged as one of the leading causes of blindness among children worldwide. ${ }^{2}$ The development of neonatal care has improved

\section{Strengths and limitations of this study}

We developed a systematic search strategy to find the best evidence to explore the relationship between sepsis and retinopathy of prematurity, and we conducted a quality assessment of each of the included studies.

- The Grading of Recommendations Assessment, Development and Evaluation approach was used to evaluate the quality of the evidence.

- All included studies were observational studies; therefore, causality was difficult to establish.

- Multiple testing due to the number of subgroup analyses could have led to false-positive results; therefore, the final results need to be interpreted with caution.

the survival rate of extremely preterm infants, but it has also increased the incidence of ROP. In a prospective study conducted by Al-Essa et $a l,{ }^{3} 64.5 \%$ of preterm infants with birth weight $(\mathrm{BW})<1501 \mathrm{~g}$ developed ROP. In a retrospective study in Saudi Arabia, ${ }^{2}$ ROP was diagnosed in $56 \%$ of infants with gestational age (GA) $<36$ weeks, and $15 \%$ of those patients were in stage 3 of the disease (severe ROP). In a population-based study in Sweden $^{4}, 34.5 \%$ of infants with GA $<27$ weeks had severe ROP, and only $19.3 \%$ of those affected infants were treated. Different incidence rates have been reported in research from Australia, ${ }^{5}$ Canada, ${ }^{6}$ Japan $^{7}$ and China. ${ }^{8}$ ROP continues to be a challenge to neonatologists and ophthalmologists, and it is vital to screen for the causes of ROP in a timely manner.

Neonatal sepsis is defined as a blood infection that occurs in infants under 90 days old, including bacterial and fungal infections. Early-onset sepsis occurs in the first week after birth, whereas late-onset sepsis occurs after 1 week and before 3 months of age. Neonatal sepsis has non-specific signs and symptoms that contribute to morbidity and mortality among preterm infants. A recent systematic review estimated that there were 
3.0 million cases of sepsis in neonates around the world, with mortality ranging from $11 \%$ to $19 \% .{ }^{9}$ Sepsis not only poses a direct risk of death but also damages organs and tissues in neonates.

Many studies have demonstrated that sepsis has a close relationship with the occurrence of ROP. According to Al-Essa $e t a l l^{3}$ the incidence of ROP in preterm infants with sepsis was 3.5 times as high as in those without sepsis $(\mathrm{OR}=3.50,95 \% \mathrm{CI} 1.02$ to 12.2$)$. Araz $e t a l^{10}$ found that sepsis was independently associated with the development of severe ROP in infant inpatients ( $\mathrm{OR}=6.86,95 \%$ CI 3.14 to 14.99). Thomas et at reported analogous results in their study $(\mathrm{OR}=1.35,95 \%$ CI 1.16 to 1.67$)$. However, Sabzehei et $a l^{11}$ and Lin $e t a l^{12}$ concluded that sepsis status was not significantly different between premature infants with and without threshold ROP ( $\mathrm{p}>0.05)$.

Considering the severe outcome of ROP and the high morbidity of sepsis, we sought to address the disagreement of several previous studies in a larger pooled sample size by conducting this meta-analysis to explore the link between sepsis and ROP.

\section{METHODS}

According to the Preferred Reporting Items for Systematic Reviews and Meta-Analyses (PRISMA), ${ }^{13}$ we developed a prospective protocol that detailed our research process, including the objectives, literature search strategies, inclusion and exclusion criteria, primary outcome and data analysis. This study adheres to PRISMA guidelines, and the PRISMA checklist is shown in online supplementary table $\mathrm{S} 1$.

\section{Literature search strategy}

We searched PubMed, the Cochrane Library and Embase for studies published from 1 January, 2000, to 1 January, 2018, using a systematic search strategy that removed duplicate titles. The detailed search strategy for this study can be found in the online supplementary table S2. The search terms used the keywords of ['sepsis' OR 'neonatal sepsis' OR 'septic' OR 'septicemia' OR 'risk factors'] AND ['premature retinopathy' OR 'ROP']. We used a combination of free words and Medical Subject Headings (MeSH) terms to search, and the final search strategy was determined by multiple pre-searches without language restrictions. Moreover, we manually searched for some eligible original documents, reviews and related articles to compensate for the deficiency of the electronic search. If multiple studies reported the same results, the latest and most complete study was selected.

\section{Study selection and data extraction}

The inclusion criteria for the literature included the following: (1) Original study involving the relationship between sepsis and ROP. (2) Diagnosis of ROP based on an ophthalmoscopic examination performed by ophthalmologists or neonatologists. (3) GA at birth $<37$ weeks. (4) Provision of original data such as the OR and 95\% CI or data that can be converted into the OR and $95 \%$ CI. (5) Total sample size $>50$. The exclusion criteria were as follows: (1) No distinction between the absence of ROP and mild ROP. (2) Duplicated reports, review articles, animal studies and editorial letters. Two reviewers (XFW and KT) independently screened the search results and determined the final list of studies to be included. If there were disagreements between their decisions, a third reviewer (HLX) made the final decision. The information we extracted for each study included the first author, year of publication, country of origin, inclusion criteria of participants, sample size, study design, literature quality score and confounding factors controlled.

\section{Quality assessment}

We used the Newcastle-Ottawa Scale (NOS), recommended by the Cochrane Manual, to evaluate the quality of the included studies. ${ }^{14}$ This scale consists of three main components: selection of study subjects, comparison between study groups and evaluation of outcomes. ${ }^{15}$ The maximum total score of this scale is nine points, and studies scoring six points or above are considered high quality. XFW and KT independently scored all items, and HLX acted as the arbiter if there were disagreements.

\section{Outcomes}

The outcome in this meta-analysis was the association between sepsis and ROP, and ROP was classified as any stage of ROP (stages 1 to 5) and severe stage of ROP (stages 3 to 5) according to the data provided by the studies. The association between sepsis and ROP was expressed as the OR of sepsis associated with ROP. We obtained the ORs and corresponding 95\% CIs from most of the included studies. When those values were not directly available, we calculated the OR and 95\% CI using the coefficient estimates and standard errors mentioned in the text.

\section{Statistical analysis}

This meta-analysis was conducted using Stata/SE 12.0 (College Station, Texas: StataCorp LP). Outcomes were assessed by pooled OR among the cohort studies, and the corresponding $95 \%$ CIs were calculated by a fixed-effects model or a random-effects model. Heterogeneity between studies was explored by Cochran's $Q$ test and the $I^{2}$ statistic, where $\mathrm{p}<0.1$ or $\mathrm{I}^{2}>50 \%$ was considered evidence of statistical heterogeneity, and we used a random-effects model for subsequent analysis. In contrast, $p>0.1$ or $\mathrm{I}^{2}<50 \%$ was considered low heterogeneity, and a fixed-effects model was used. Predefined subgroup analyses were conducted to explore the sources of heterogeneity and to obtain the adjusted ORs by GA ( $\leq 34$ weeks or $<37$ weeks), BW $(\leq 1.5 \mathrm{~kg}$ or $\leq 2.0 \mathrm{~kg})$, study location (Asia, Africa, USA or Europe), study design (prospective or retrospective) and year of publication (2000 2010 or 2011 2018). Potential publication bias was detected by the combined use of funnel plots and Egger's test. We also conducted a sensitivity analysis by assessing the changes in the OR and $95 \% \mathrm{CI}$ after removing any single study. The OR we 
selected was the adjusted $\mathrm{OR}$, and $\mathrm{p}<0.05$ was considered statistically significant.

\section{Grading the evidence}

Based on the outcomes of the systematic review, XFW and KT independently using the Grading of Recommendations Assessment, Development and Evaluation (GRADE) approach to determine the quality of the evidence. ${ }^{16}$ The quality of the evidence was rated as high, moderate, low or very low. Since all of the studies included in this meta-analysis were observational studies (including cohort and case-control studies), the quality of the evidence was initially defined as low. The rating can be decreased to very low if serious or very serious issues are noted in connection with risk of bias, inconsistency of results, indirectness of evidence, imprecision or publication bias. However, upgrading may be warranted in observational studies if the magnitude of the treatment effect is large (Relative Risk $(\mathrm{RR})>2$ or $\mathrm{RR}<0.5$ ) or very large ( $R R>5$ or $R R<0.2)$, if there is evidence of a dose-response relation or if all plausible biases would decrease the magnitude of an apparent treatment effect. Therefore, the quality of the evidence can range from very low to high.

\section{Patient and public involvement}

Our research is based on previously published studies. Patients were not directly involved in the design, implementation or analysis of this study. We will disseminate our findings through the journal's public access.

\section{RESULTS}

\section{Search results}

Our preliminary search located 509 search records of candidate studies. After browsing the title, abstract and full text, we identified a total of 16 eligible studies with full text and entered them into the data analysis (figure 1).

\section{Characteristics and quality of the included studies}

The characteristics of the included studies were shown in table 1. These studies were published from 2000 to 2018 and came from five continents: seven studies were from Asia, four from USA, two each from Africa and Europe and one from Oceania. Of the studies, 11 explored the association between sepsis and any stage of ROP, and six examined the relationship between sepsis and severe stage of ROP. The sample sizes in these studies varied from 110 to 5718. The participants were mainly from the intensive care units, neonatal intensive care units and neonatal centres of local hospitals. The inclusion criteria for premature infants varied considerably: the GA criteria ranged from $<28$ weeks to $<37$ weeks, and only 12 studies provided the specific BW range. Most included studies reported the multivariable-adjusted OR and corresponding 95\% CI, although one study provided only univariate analysis outcomes. ${ }^{17}$ There were eight prospective cohort studies, seven retrospective cohort studies and

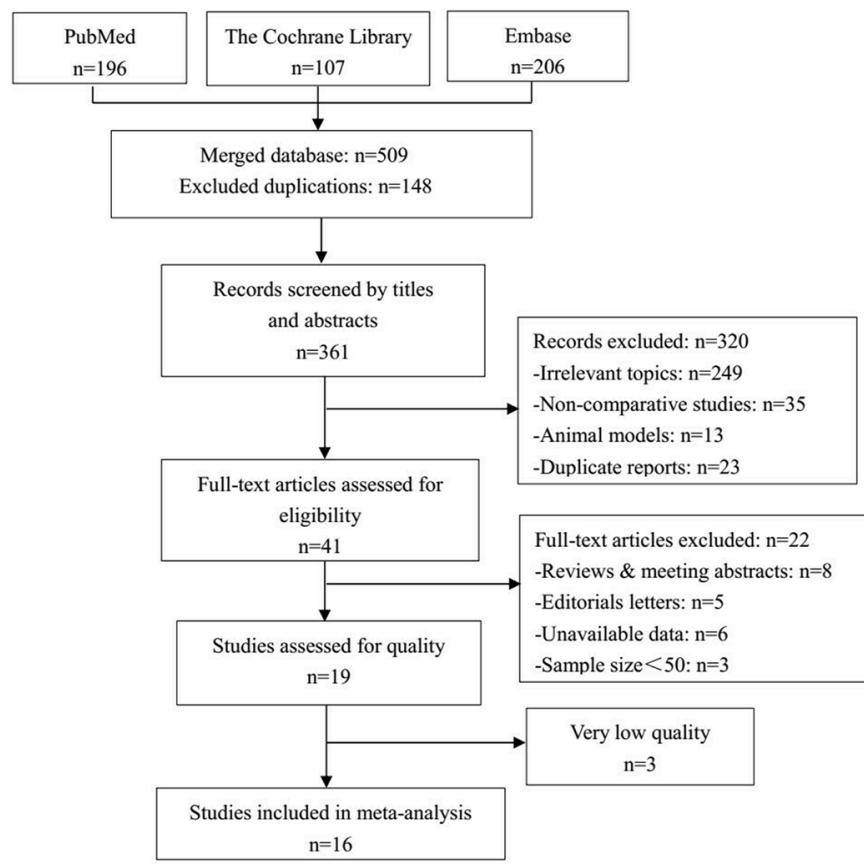

Figure 1 Flow diagram of included/excluded studies. A total of 509 documents were found in the initial search. After removing duplicates, reading abstracts and full texts and evaluating the quality of the articles, 16 eligible studies were included and analysed.

one nested case-control study, which also came from an institutional retrospective cohort. All included studies had reasonably well-matched groups that appeared to represent the same populations. Fifteen studies provided confounding factors that were controlled, 13 studies reported adequate follow-up to observe the occurrence of the disease and nine studies reported the rate of follow-up or loss to follow-up. The NOS scores of these studies ranged from 5 to 9 . A total of 15 studies could be identified as high-quality studies because they scored 6 points or above. Agreement between the two reviewers was $95 \%$ for study selection and $93 \%$ for quality assessment. Detailed information on the quality assessment is shown in online supplementary table S3.

\section{Sepsis and any stage of ROP}

Eleven studies involving a total of 5388 participants reported adjusted ORs for association between sepsis and any stage of ROP. Considering all these adjusted ORs combined, sepsis was associated with a significant risk of any ROP among premature infants in the random-effects model ( $\mathrm{OR}=1.57,95 \%$ CI 1.31 to 1.89$)$. Moderate heterogeneity was detected $\left(\mathrm{I}^{2}=56.3 \%, \mathrm{p}=0.011\right)$ (figure 2 ).

\section{Sepsis and severe stage of ROP}

Six studies including a total of 7660 premature infants provided ORs for the severe stage of ROP and were combined in this meta-analysis. Figure 3 shows the combined effect from the random-effects model. The pooled OR was 2.33 (95\% CI 1.21 to 4.51 ), with evidence of substantial heterogeneity $\left(\mathrm{I}^{2}=81.8 \%, \mathrm{p}<0.001\right)$. Both 


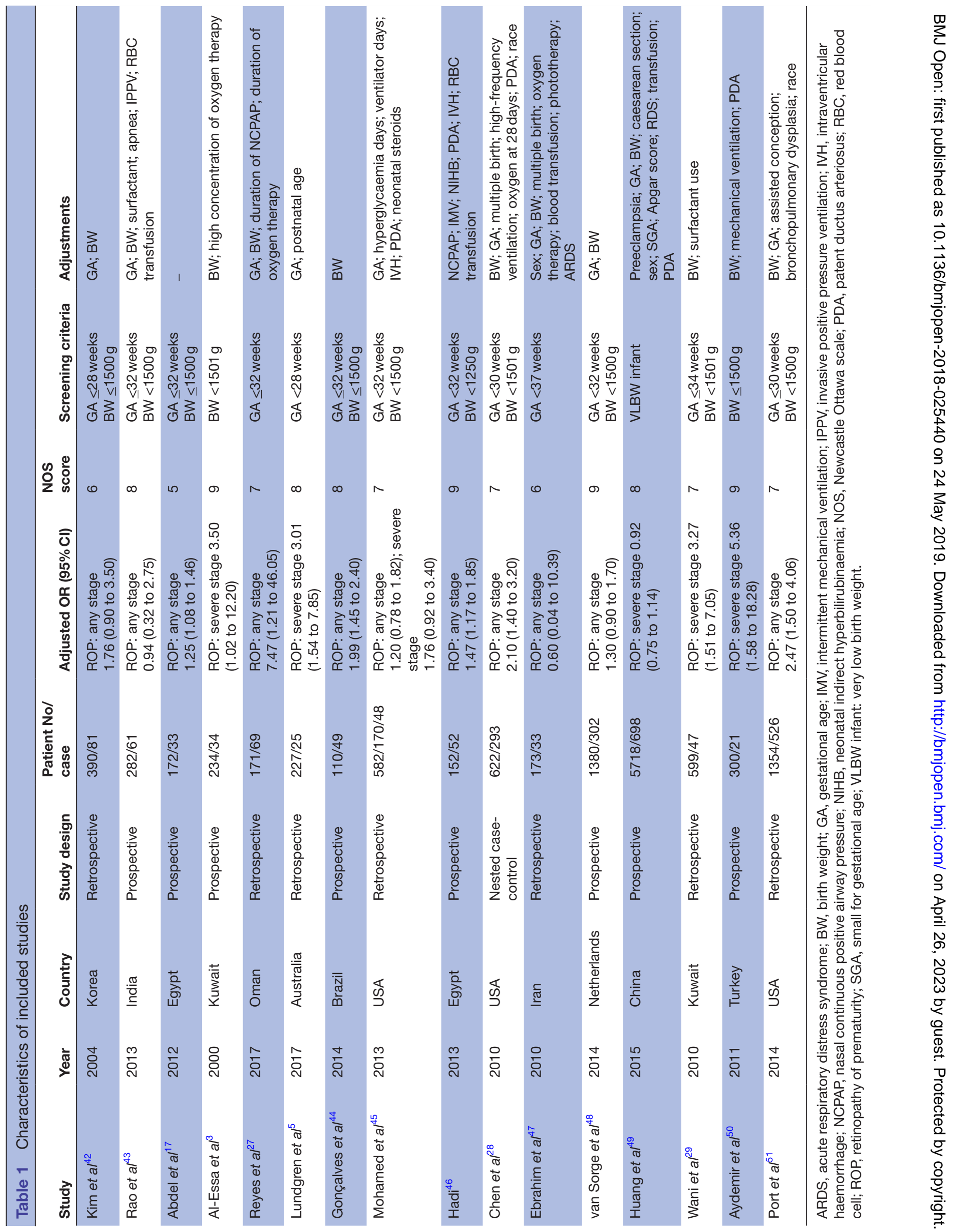




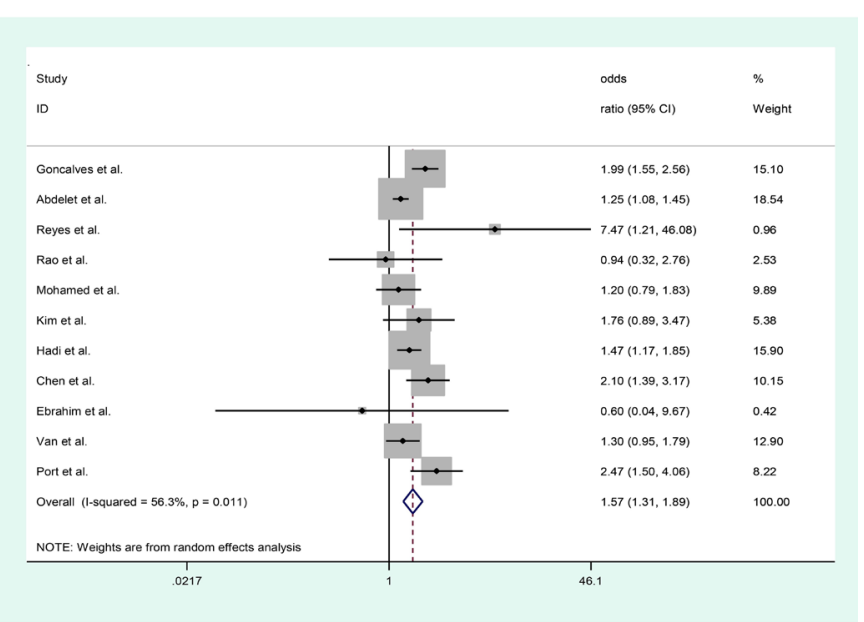

Figure 2 Forest plot of the association between sepsis and any stage of ROP. $n=5388$. Data were expressed as ORs and corresponding 95\% Cls. Pooled effect evaluation, represented by a diamond, was obtained with a randomeffects model. The heterogeneity between studies was explored by Cochran's $Q$ test and the $\mathrm{I}^{2}$ statistic, where $\mathrm{p}<0.1$ or $\mathrm{I}^{2}>50 \%$ was considered evidence of substantial heterogeneity. ROP, retinopathy of prematurity.

results demonstrated that sepsis was closely related to the development of ROP in premature infants.

\section{Subgroup analysis}

As shown in table 2, in any stage of ROP, the heterogeneity between GA, BW and geographical subgroups was significantly less than their combined effects, respectively, suggesting that these categories could explain the potential heterogeneity. GA $<34$ weeks (OR=1.70, 95\% CI 1.39 to 2.70$)$, and $\mathrm{BW}<1.5 \mathrm{~kg}(\mathrm{OR}=1.66,95 \% \mathrm{CI} 1.38$ to 2.01$)$ showed an elevated risk in any stage of ROP. Research

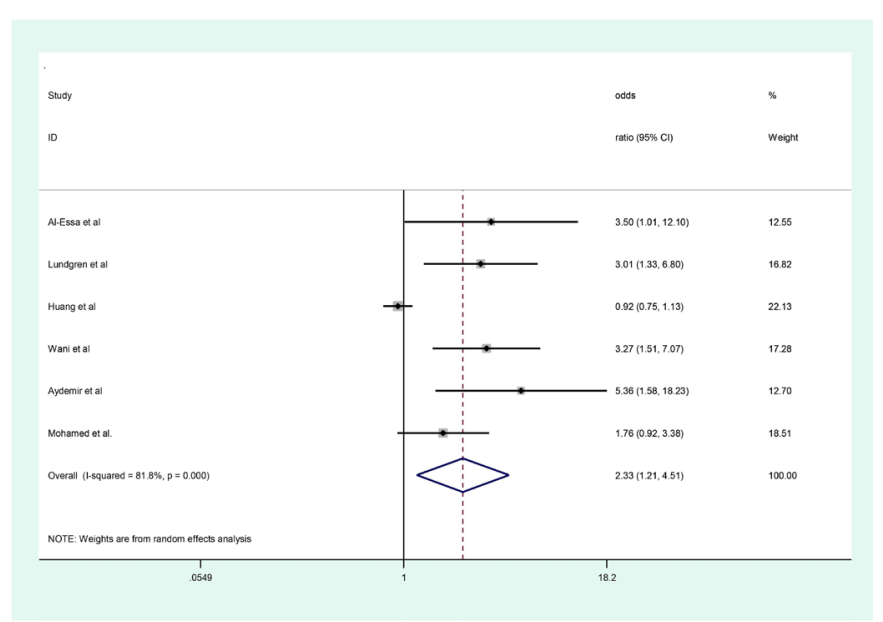

Figure 3 Forest plot of the association between sepsis and severe stage of ROP. $n=7660$. Data were expressed as ORs and corresponding 95\% Cls. Pooled effect evaluation, represented by a diamond, was obtained with a randomeffects model. The heterogeneity between studies was explored by Cochran's $Q$ test and the $\mathrm{I}^{2}$ statistic, where $\mathrm{p}<0.1$ or $\mathrm{I}^{2}>50 \%$ was considered evidence of substantial heterogeneity. ROP, retinopathy of prematurity. published in 2000 2010 (OR=1.96, 95\% CI 1.38 to 2.79) and studies conducted in the USA also showed an increased risk of any ROP (OR=1.87, 95\% CI 1.43 to 2.45$)$. Moderate heterogeneity was found in the prospective cohort studies $\left(\mathrm{I}^{2}=62.1 \%, \mathrm{p}=0.032\right)$, and studies published in 2011 2018 were responsible for most of the heterogeneity $\left(\mathrm{I}^{2}=63.4 \%, \mathrm{p}=0.008\right)$. The results for severe stages of ROP were different, and it was difficult to identify sources of heterogeneity, since there were few studies that could be combined.

\section{Publication bias and sensitivity analysis}

There was no evidence of asymmetry in the funnel plots (figure 4), and Egger's test showed $1.12(\mathrm{p}=0.290)$. Considering the funnel plots and Egger's test, we concluded that there was no apparent publication bias among the studies that examined all stages of ROP. However, publication bias was not assessed in studies on the severe stage of ROP because there was insufficient power to evaluate funnel plot asymmetry and small-study effects $(<10$ studies included).

When we excluded Abdelet's study, which had the lowest NOS score, the pooled OR was 1.66 (95\% CI 1.37 to 2.01) in any stage of ROP. When we removed any single study in any stage of ROP and severe stage of ROP, the pooled ORs ranged from 1.50 (95\% CI 1.25 to 1.79 ) to 1.66 (95\% CI 1.37 to 2.01) and from 2.20 (95\% CI 1.09 to 4.45$)$ to 2.74 (95\% CI 1.87 to 4.02 ), respectively. No obvious change was found in the pooled ORs, and each was statistically significant.

\section{GRADE assessment}

The quality of evidence was rated as low for the outcomes of association between sepsis and any stage of ROP, having been upgraded by one point because all plausible biases would decrease the magnitude of an apparent treatment effect, and been downgraded because moderate heterogeneity between studies was detected $\left(\mathrm{I}^{2}=56.3 \%, \mathrm{p}=0.011\right)$ and could not be fully explained. Meanwhile, the quality of the evidence was also rated as low for the relationship between sepsis and severe stage of ROP: the magnitude of the effect was large (OR $>2$ based on consistent evidence from at least two studies, with no plausible confounders), which added one point, but there was evidence of substantial heterogeneity between studies that could not be explained $\left(\mathrm{I}^{2}=81.8 \%, \mathrm{p}<0.001\right)$, which subtracted one point, leaving the final score at two points for a quality level of 'low'. A summary of the GRADE assessment is shown in online supplementary table S4.

\section{DISCUSSION}

The prevalence of ROP and its association with sepsis are great challenges worthy of global attention. To our knowledge, this is the first meta-analysis to assess the independent risk of sepsis in ROP. Our research applied strict inclusion criteria. According to the International Classification of Retinopathy of Prematurity, ${ }^{18}$ ROP is divided 
Table 2 Subgroup meta-analysis and analysis of heterogeneity

\begin{tabular}{|c|c|c|c|c|c|}
\hline Subgroup & $\begin{array}{l}\text { Number of } \\
\text { studies }\end{array}$ & $\begin{array}{l}\text { Patient } \\
\text { No/case }\end{array}$ & Adjusted OR $(95 \% \mathrm{Cl})$ & $\mathrm{I}^{2}(\%)$ & $P$ value \\
\hline \multicolumn{6}{|l|}{ Any stage of ROP } \\
\hline \multicolumn{6}{|l|}{ Gestational age } \\
\hline$\leq 34$ weeks & 8 & $4761 / 1542$ & 1.70 (1.39 to 2.07$)$ & 49.8 & $<0.001$ \\
\hline$<37$ weeks & 3 & $627 / 127$ & 1.24 (1.07 to 1.44$)$ & 0 & 0.005 \\
\hline \multicolumn{6}{|l|}{ Birth weight } \\
\hline$\leq 1.5 \mathrm{~kg}$ & 7 & $4590 / 1473$ & 1.66 (1.38 to 2.01$)$ & 46.9 & $<0.001$ \\
\hline$\leq 2.0 \mathrm{~kg}$ & 4 & $798 / 196$ & 1.34 (0.75 to 2.40$)$ & 29.3 & 0.323 \\
\hline \multicolumn{6}{|c|}{ Geographical location } \\
\hline Asian & 4 & $1016 / 244$ & 1.66 (0.79 to 3.49$)$ & 29.2 & 0.183 \\
\hline Africa & 2 & $324 / 85$ & 1.32 (1.14 to 1.54$)$ & 25.5 & $<0.001$ \\
\hline USA & 4 & $2668 / 1038$ & 1.87 (1.43 to 2.45$)$ & 49.5 & $<0.001$ \\
\hline Europe & 1 & $1380 / 302$ & 1.30 (0.95 to 1.79$)$ & - & 0.106 \\
\hline \multicolumn{6}{|l|}{ Study design } \\
\hline Prospective & 5 & $2096 / 497$ & 1.45 (1.18 to 1.77$)$ & 62.1 & $<0.001$ \\
\hline Retrospective & 6 & $3292 / 1172$ & 1.86 (1.32 to 2.64$)$ & 41.5 & $<0.001$ \\
\hline \multicolumn{6}{|l|}{ Year of publication } \\
\hline 2000 2010 & 3 & $1185 / 407$ & 1.96 (1.38 to 2.79$)$ & 0 & $<0.001$ \\
\hline 2011 2018 & 8 & $4203 / 1262$ & 1.52 (1.24 to 1.86$)$ & 63.4 & $<0.001$ \\
\hline \multicolumn{6}{|l|}{ Severe stage of ROP } \\
\hline \multicolumn{6}{|l|}{ Gestational age } \\
\hline$\leq 34$ weeks & 5 & $7426 / 839$ & 2.20 (1.09 to 4.45$)$ & 83.7 & 0.028 \\
\hline$<37$ weeks & 1 & $234 / 34$ & 3.50 (1.01 to 12.10$)$ & - & 0.048 \\
\hline \multicolumn{6}{|c|}{ Geographical location } \\
\hline Asian & 3 & $6551 / 779$ & 1.99 (0.70 to 5.67$)$ & 85.2 & 0.196 \\
\hline Oceania & 1 & $227 / 25$ & 3.01 (1.33 to 6.80$)$ & - & 0.008 \\
\hline Europe & 1 & $300 / 21$ & 5.36 (1.58 to 18.23$)$ & - & 0.007 \\
\hline USA & 1 & $582 / 48$ & 1.76 (0.92 to 3.38$)$ & - & 0.090 \\
\hline \multicolumn{6}{|l|}{ Study design } \\
\hline Prospective & 3 & $4062 / 1578$ & 2.30 (0.65 to 8.14$)$ & 83.0 & 0.195 \\
\hline Retrospective & 3 & $1408 / 120$ & 2.46 (1.61 to 3.76$)$ & 0 & $<0.001$ \\
\hline \multicolumn{6}{|l|}{ Year of publication } \\
\hline $2000 \sim 2010$ & 2 & $833 / 81$ & 3.33 (1.73 to 6.41$)$ & 0 & $<0.001$ \\
\hline 2011 2018 & 4 & $6827 / 792$ & 1.98 (0.93 to 4.23$)$ & 82.4 & 0.078 \\
\hline
\end{tabular}

ROP, retinopathy of prematurity.

into 5 stages, of which stages 1 to 2 are defined as mild ROP and stages 3 to 5 are defined as severe ROP. The former does not require treatment, while the latter does. ${ }^{10}$ To ensure the homogeneity of these studies, we included only those that compared a group without ROP to a group with ROP, with or without restrictions on severity. Any study that was divided into 'no treatment' (no ROP and mild ROP) versus 'treatment' (severe ROP) was excluded. After controlling for confounding factors such as GA and BW and adopting adjusted ORs, we speculated that sepsis distinctly increases the overall risk of ROP and the risk of severe ROP among preterm infants.

How does sepsis induce ROP or increase its severity? Successful trials have yielded some possible explanations, which can be summarised as follows: (1) The pathogenical microorganisms and their toxins cause sepsis-related damage in vascular endothelial cells, making white blood cells readily adhere to the blood vessel walls and form microthrombi in the small blood vessels of the retina; these microthrombi lead to blood vessel obstruction 


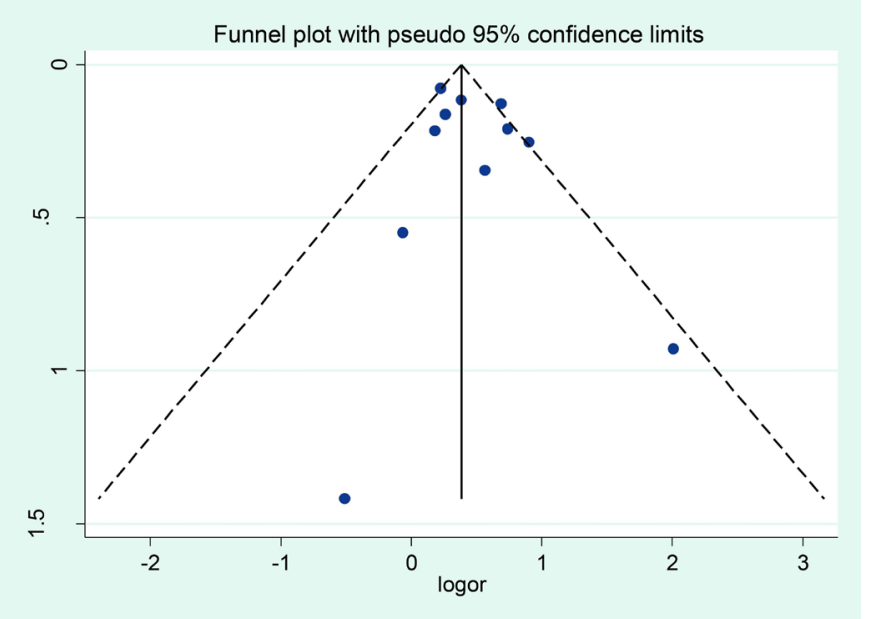

Figure 4 Funnel plots of the association between sepsis and any stage of ROP. The solid line represents the overall pooled estimate for all included studies. Dashed lines represent $95 \%$ Cls. Egger's test gave a result of 0.20 , $\mathrm{p}=0.06$. ROP, retinopathy of prematurity.

and increased permeability. Eventually, an area of retinal non-perfusion forms, or a previously formed area expands. ${ }^{19}$ (2) Sepsis often aggravates the body's oxidative stress response, and small doses of oxidative stress products transmit signals through vascular endothelial growth factor-2 to vascular endothelial cells, promoting vascular endothelial cell proliferation and migration. Furthermore, a large dose of oxidative stress products from lipid peroxidation of biofilm will damage the biofilm, leading to cell degeneration and necrosis, ${ }^{20}$ thereby aggravating retinal and vascular lesions. (3) The effects of inflammatory mediators and growth factors such as interleukin-1 $\beta$ can significantly increase the activity of hypoxia-inducible factor $(\mathrm{HIF}-1 \alpha),{ }^{21}$ and transforming growth factor- $\beta$ can inhibit the degradation of HIF-1 $\alpha$ through the Smad signalling pathway. ${ }^{22}$ Finally, ROP is induced and exacerbated by the HIF-l $\alpha$ pathway. In addition, there may be other inflammatory factors, such as phospholipase-2 and prostaglandins, that affect retinal neovascularization. ${ }^{23}$

Although the final combined value was statistically significant, our estimates may have some potential bias due to the inconsistent control of confounding factors in the original studies. Most studies controlled the GA and BW of preterm infants at birth, and some studies regarded the duration of oxygen therapy, blood transfusion and patent ductus arteriosus as confounding factors to control. However, few studies evaluated maternal factors, such as maternal age, hypertension, antepartum haemorrhage and premature rupture of membranes, in the relationship between sepsis and ROP. Yang et $a t^{24}$ found that very low birth weight (VLBW) infants whose mothers had preeclampsia faced an important increase in the risk of ROP. Darlow et $a l^{25}$ and Bardin et $a l^{26}$ reported that the changes in the hormonal milieu of the maternal uterus and the series of problems it raises, including chronic intrauterine hypoxia, antioxidant deficiency and abnormal growth factor levels, might also contribute to the increased risk of ROP in preterm infants. These factors have not been taken into account in the studies included in this review; this omission from the original studies may cause our results to deviate from the real effects. In addition, a total of 12 studies in this meta-analysis included premature infants with BW less than $1500 \mathrm{~g}$. Therefore, the combined effect value may represent this type of premature infant, not all premature infants.

As mentioned above, sepsis can be classified as early or late and as bacterial or fungal. Since most of the original studies performed fundus examinations for ROP screening 4 6 weeks after birth, only two studies ${ }^{27} 28$ provided findings that might include late-onset sepsis. It is difficult for us to distinguish whether these patients had early-onset or late-onset sepsis. Moreover, all studies showed that the diagnosis of sepsis was based on clinical blood culture, but they did not make a clear distinction between the pathogens. Two studies mentioned sepsis in general terms that encompassed both fungal sepsis and bacterial sepsis. ${ }^{2829}$ Therefore, we have not yet been able to combine the ORs of sepsis with different stages and different pathogens. Hussain et al found that culture-positive sepsis of any aetiology was significantly associated with ROP. ${ }^{30}$ Manzoni et al found that fungal (but not bacterial) sepsis was independently associated with ROP, but only in VLBW neonates and only with threshold ROP. ${ }^{31}$ Bharwani also reported that systemic fungal infection (SFI) was associated with the development of all degrees of ROP in VLBW infants. ${ }^{32}$ However, the current studies and review do not address the mechanism of the association between SFI and ROP or prove any cause and effect; therefore, this association needs to be studied in a larger population before any conclusions can be drawn.

In a subgroup analysis, we found that infants born with shorter GA and lighter BW were more likely to develop ROP. This finding is consistent with the results of most previous studies, which confirmed that short GA and low BW were independent risk factors for ROP, especially for VLBW infants. ${ }^{33-37}$ Some researchers even believed that BW might be a more important criterion than GA for the screening of ROP. ${ }^{38}$ Preterm infants, regardless of GA or whether their BW was below the normal range, were much more susceptible to infection than full-term babies and often lacked the capacity to overcome it, which could induce or exacerbate ROP in the event of a severe infection that leads to sepsis. ${ }^{39}$ In addition, due to the incomplete development of retinal blood vessels in preterm infants, it is easy to induce the formation of new retinal blood vessels and fibrous tissue, which may be a very common cause of ROP.

This study also showed regional differences in the relationship between sepsis and any stage of ROP, with the risk being highest in the USA, second highest in Asia and lowest in Europe, with Africa having a slightly higher risk than Europe. This phenomenon might be associated with ethnicity. Aralikatti et al reported that Asian and Black premature infants had a higher risk of developing 
threshold ROP compared with White infants, ${ }^{40}$ and similar outcomes were reported by $\mathrm{Ng}$ et $a l^{41}$ The former believe that the differential risk may be related to pigmentation, because animal models of ROP have shown that pigmented strains are more susceptible to ischaemia-induced retinal neovascularization than albino strains. The latter group holds that this difference was because of the better survival of Asian infants, as preterm infants in these ethnic groups are more likely to survive and hence develop severe ROP than preterm infants of other ethnicities. Both groups agreed that Asian and African premature infants needed more treatment than White infants. However, according to Darlow et $a l^{25}$ premature infants born to Asian mothers had higher risk than White infants, but this difference did not exist in the adjusted analysis. Chen $e t a l^{28}$ also found that there was no significant association between ROP and ethnicity. Thus, further evidence is needed to explain the specific mechanisms and causes of the different rates of ROP prevalence among different ethnic groups.

The overall quality of the evidence was assessed by the GRADE approach, which showed low quality for the relationship between sepsis and both any stage of ROP and severe stage of ROP; thus, we have limited confidence in the estimation of both effect value. Since few studies could be combined for severe ROP, some potential publication bias may have gone undetected and caused the level of evidence to be overestimated. Further study is likely or very likely to have an important impact on our confidence in the estimated effect and may change our estimate.

This meta-analysis has the following limitations. First, the number of studies included is limited, and all original articles are observational studies, from which causality can rarely be established. Second, different inclusion criteria in each study and inaccurately specified criteria in a few studies might have influenced the pooled effect. Third, multiple testing due to the number of subgroup analyses could have led to false-positive results. Finally, although we did not use language restrictions in the search strategy, the final articles included were mainly in English. These limitations must be taken into account when evaluating the final outcomes.

\section{CONCLUSION}

Our meta-analysis shows that sepsis is closely related to the development of any stage of ROP and severe stage of ROP. This provides valuable information for neonatologists and ophthalmologists regarding the treatment of preterm infants. However, considering the shortcomings of this study, our results need to be interpreted cautiously. More evidence is needed to validate our findings and provide constructive advice for clinical practice, and well-designed trials are needed to provide a clearer explanation of the mechanisms and interrelationships between ROP and different types of sepsis. Strengthening the screening and intervention process for sepsis in preterm infants and preventing them from developing blindness is a global priority that depends on the joint efforts of researchers and clinicians worldwide.

Contributors XFW and HLX designed the study and the search strategy. XFW and KT performed the study selection and quality assessment. LC and SXC performed the data analysis. XFW wrote the first draft. HLX critically revised the manuscript and approved the final version as submitted.

Funding The authors have not declared a specific grant for this research from any funding agency in the public, commercial or not-for-profit sectors.

Competing interests None declared.

Patient consent for publication Not required.

Provenance and peer review Not commissioned; externally peer reviewed. Data sharing statement No additional data are available.

Open access This is an open access article distributed in accordance with the Creative Commons Attribution Non Commercial (CC BY-NC 4.0) license, which permits others to distribute, remix, adapt, build upon this work non-commercially, and license their derivative works on different terms, provided the original work is properly cited, appropriate credit is given, any changes made indicated, and the use is non-commercial. See: http://creativecommons.org/licenses/by-nc/4.0/.

\section{REFERENCES}

1. Abrishami M, Maemori GA, Boskabadi $\mathrm{H}$, et al. Incidence and risk factors of retinopathy of prematurity in mashhad, northeast iran. Iran Red Crescent Med J 2013;15:229-33.

2. Binkhathlan AA, Almahmoud LA, Saleh MJ, et al. Retinopathy of prematurity in Saudi Arabia: incidence, risk factors, and the applicability of current screening criteria. $\mathrm{Br} J$ Ophthalmol 2008;92:167-9.

3. Al-Essa M, Azad RV, Rashwan N. Threshold stage of retinopathy of prematurity: maternal and neonatal risk factors. Ann Saudi Med 2000;20:129-31.

4. Stoltz Sjöström E, Lundgren P, Öhlund I, et al. Low energy intake during the first 4 weeks of life increases the risk for severe retinopathy of prematurity in extremely preterm infants. Arch Dis Child Fetal Neonatal Ed 2016;101:F108-F13.

5. Lundgren P, Athikarisamy SE, Patole S, et al. Duration of anaemia during the first week of life is an independent risk factor for retinopathy of prematurity. Acta paediatrica 2017.

6. Thomas K, Shah PS, Canning R, et al. Retinopathy of prematurity: Risk factors and variability in Canadian neonatal intensive care units. J Neonatal Perinatal Med 2015;8:207-14.

7. Enomoto H, Miki A, Matsumiya W, et al. Evaluation of oxygen supplementation status as a risk factor associated with the development of severe retinopathy of prematurity. Ophthalmologica 2015;234:135-8.

8. Li WL, He L, Liu XH, et al. Analysis of risk factors for retinopathy of prematurity. Int J Ophthalmol 2011;4:631-3.

9. Fleischmann-Struzek C, Goldfarb DM, Schlattmann P, et al. The global burden of paediatric and neonatal sepsis: a systematic review. Lancet Respir Med 2018;6:223-30.

10. Araz-Ersan B, Kir N, Akarcay K, et al. Epidemiological analysis of retinopathy of prematurity in a referral centre in Turkey. The British journal of ophthalmology 2013;97:15-17.

11. Sabzehei MK, Afjeh SA, Dastjani Farahani A, et al. Retinopathy of prematurity: incidence, risk factors, and outcome. Arch Iran Med 2013;16:507-12.

12. Lin HJ, Lin CC, Tsai SW, et al. Risk factors for retinopathy of prematurity in very low birth-weight infants. J Chin Med Assoc 2003;66:662-8.

13. Liberati A, Altman DG, Tetzlaff J, et al. The PRISMA statement for reporting systematic reviews and meta-analyses of studies that evaluate healthcare interventions: explanation and elaboration. BMJ 2009;339:b2700.

14. Margulis AV, Pladevall M, Riera-Guardia N, et al. Quality assessment of observational studies in a drug-safety systematic review, comparison of two tools: the Newcastle-Ottawa Scale and the RTI item bank. Clin Epidemiol 2014;6:359-68.

15. Fan X, Lin T, Xu K, et al. Laparoendoscopic single-site nephrectomy compared with conventional laparoscopic nephrectomy: a systematic review and meta-analysis of comparative studies. Eur Urol 2012;62:601-12. 
16. Guyatt GH, Oxman AD, Vist GE, et al. GRADE: an emerging consensus on rating quality of evidence and strength of recommendations. BMJ 2008;336:924-6.

17. Abdel HA, Mohamed GB, Othman MF. Retinopathy of Prematurity: A Study of Incidence and Risk Factors in NICU of Al-Minya University Hospital in Egypt. J Clin Neonatol 2012;1:76-81.

18. Quinn G, International Committee for the Classification of Retinopathy of Prematurity. The International Classification of Retinopathy of Prematurity revisited. Arch Ophthalmol 2005;123:991-9.

19. Joussen AM, Poulaki V, Le ML, et al. A central role for inflammation in the pathogenesis of diabetic retinopathy. Faseb J 2004;18:1450-2.

20. Ushio-Fukai M. VEGF signaling through NADPH oxidase-derived ROS. Antioxid Redox Signal 2007;9:731-9.

21. Hellwig-Bürgel T, Rutkowski K, Metzen E, et al. Interleukin-1 beta and tumor necrosis factor-alpha stimulate DNA binding of hypoxiainducible factor-1. Blood 1999;94:1561-7.

22. McMahon S, Charbonneau M, Grandmont S, et al. Transforming growth factor beta1 induces hypoxia-inducible factor-1 stabilization through selective inhibition of PHD2 expression. J Biol Chem 2006:281:24171.

23. Hardy P, Beauchamp M, Sennlaub F, et al. New insights into the retinal circulation: inflammatory lipid mediators in ischemic retinopathy. Prostaglandins Leukot Essent Fatty Acids 2005;72:301-25.

24. Yang CY, Lien R, Yang PH, et al. Analysis of incidence and risk factors of retinopathy of prematurity among very-low-birth-weight infants in North Taiwan. Pediatr Neonatol 2011;52:321-6.

25. Darlow BA, Hutchinson JL, Henderson-Smart DJ, et al. Prenatal risk factors for severe retinopathy of prematurity among very preterm infants of the Australian and New Zealand Neonatal Network. Pediatrics 2005;115:990-6.

26. Bardin C, Zelkowitz P, Papageorgiou A. Outcome of small-forgestational age and appropriate-for-gestational age infants born before 27 weeks of gestation. Pediatrics 1997;100:E4.

27. Reyes ZS, Al-Mulaabed SW, Bataclan F, et al. Retinopathy of prematurity: revisiting incidence and risk factors from Oman compared to other countries. Oman J Ophthalmol 2017;10:26-32.

28. Chen M, Citil A, McCabe F, et al. Infection, oxygen, and immaturity: interacting risk factors for retinopathy of prematurity. Neonatology 2011;99:125-32.

29. Wani VB, Kumar N, Sabti K, et al. Results of screening for retinopathy of prematurity in a large nursery in Kuwait: Incidence and risk factors. Indian J Ophthalmol 2010;58:204-8.

30. Hussain N, Clive J, Bhandari V. Current incidence of retinopathy of prematurity, 1989-1997. Pediatrics 1999;104:e26.

31. Manzoni P, Maestri A, Leonessa M, et al. Fungal and bacterial sepsis and threshold ROP in preterm very low birth weight neonates. $J$ Perinatol 2006;26:23-30.

32. Bharwani SK, Dhanireddy R. Systemic fungal infection is associated with the development of retinopathy of prematurity in very low birth weight infants: a meta-review. J Perinatol 2008;28:61-6.

33. 최성호 함돈일. Incidence and Risk Factors of Retinopathy of Prematurity in Extremely Low Birth Weight and Very Low Birth
Weight Infants. Journal of The Korean Ophthalmological Society 2006:47:918-26.

34. Kavurt S, Yücel H, Hekimoğlu E, et al. Retinopathy of prematurity(ROP): Cases and risk factors. Cocuk Sagligi ve Hastaliklari Dergisi 2012;55:125-31.

35. Shah V, Chiang J, Chung S, et al. To determine the incidence, risk factors and need for surgery for retinopathy of prematurity(ROP) among Very-Low-Birth-Weight(VLBW) infants weighing. Archives of Disease in Childhood 2014;99:A465.

36. Akkoyun I, Oto S, Yilmaz G, et al. Risk factors in the development of mild and severe retinopathy of prematurity. J Aapos 2006;10:449-53.

37. Bas AY, Demirel N, Koc E, et al. Incidence, risk factors and severity of retinopathy of prematurity in Turkey (TR-ROP study): a prospective, multicentre study in 69 neonatal intensive care units. Br J Ophthalmol $2018 ; 102$.

38. Araz-Ersan B, Kir N, Akarcay K, et al. Epidemiological analysis of retinopathy of prematurity in a referral centre in Turkey. Br J Ophthalmol 2013;97:15-17.

39. Quiroga A, Moxon S. Preventing sight-threatening ROP: the role of nurses in reducing the risk. Community Eye Health 2017;30:53-4.

40. Aralikatti AK, Mitra A, Denniston AK, et al. Is ethnicity a risk factor for severe retinopathy of prematurity? Arch Dis Child Fetal Neonatal Ed 2010;95:F174-6.

41. Ng YK, Fielder AR, Shaw DE, et al. Epidemiology of retinopathy of prematurity. Lancet 1988;2:1235-8.

42. Kim TI, Sohn J, Pi SY, et al. Postnatal risk factors of retinopathy of prematurity. Paediatr Perinat Epidemiol 2004;18:130-4.

43. Rao KA, Purkayastha J, Hazarika M, et al. Analysis of prenatal and postnatal risk factors of retinopathy of prematurity in a tertiary care hospital in South India. Indian J Ophthalmol 2013;61:640-4.

44. Gonçalves E, Násser LS, Martelli DR, et al. Incidence and risk factors for retinopathy of prematurity in a Brazilian reference service. Sao Paulo Med J 2014;132:85-91.

45. Mohamed S, Murray JC, Dagle JM, et al. Hyperglycemia as a risk factor for the development of retinopathy of prematurity. $B M C$ Pediatr 2013;13:78.

46. Hadi AM, Hamdy IS. Correlation between risk factors during the neonatal period and appearance of retinopathy of prematurity in preterm infants in neonatal intensive care units in Alexandria, Egypt. Clin Ophthalmol 2013;7:831-7.

47. Ebrahim M, Ahmad RS, Mohammad M. Incidence and risk factors of retinopathy of prematurity in Babol, North of Iran. Ophthalmic Epidemiol 2010;17:166-70.

48. van Sorge AJ, Termote JU, Kerkhoff FT, et al. Nationwide inventory of risk factors for retinopathy of prematurity in the Netherlands. $J$ Pediatr 2014;164:494-8.

49. Huang $\mathrm{HC}$, Yang $\mathrm{HI}$, Chou $\mathrm{HC}$, et al. Preeclampsia and Retinopathy of Prematurity in Very-Low-Birth-Weight Infants: A Population-Based Study. PLoS One 2015;10:e0143248

50. Aydemir O, Sarikabadayi YU, Aydemir C, et al. Adjusted poor weight gain for birth weight and gestational age as a predictor of severe ROP in VLBW infants. Eye 2011;25:725-9.

51. Port $A D$, Chan RV, Ostmo $S$, et al. Risk factors for retinopathy of prematurity: insights from outlier infants. Graefes Arch Clin Exp Ophthalmol 2014;252:1669-77. 